\title{
Accelerating XML Structural Matching Using Suffix Bitmaps
}

\author{
Feng Shao, Gang Chen, and Jinxiang Dong \\ Dept. of Computer Science, Zhejiang University, Hangzhou, P.R. China \\ microf_shao@msn.com, \\ cg@zju.edu.cn, djx@zju.edu.cn
}

\begin{abstract}
With the rapidly increasing popularity of XML as a data format, there is a large demand for efficient techniques in structural matching of XML data. We propose a novel filtering technique to speed up the structural matching of XML data, which is based on an auxiliary data structure called suffix bitmap. The suffix bitmap captures in a packed format the suffix tag name list of the nodes in an XML document. By comparing the respective suffix bitmaps, most of the unmatched subtrees of a document can be skipped efficiently in the course of structural matching process. Using the suffix bitmap filtering, we extend two state-of-the-art structural matching algorithms: namely the traversal matching algorithm and the structural join matching algorithm. The experimental results show that the extended algorithms considerably outperform the original ones.
\end{abstract}

Keywords: XML, Suffix Bitmap, Structural Matching, Filtering.

\section{Introduction}

In the past decade, while XML has become the de facto standard of information representation and exchange over the Internet, efficient XML query processing techniques are still in great demand. The core problem of XML query processing, namely structural matching, still remains to be a great challenge. In this paper, we propose a novel acceleration technique for structural matching of XML documents. Our method utilizes an auxiliary data structure called suffix bitmap, which compresses the suffix tag names list of XML nodes in a packed format. In an XML document tree, each node corresponds to a sub-tree, which is rooted at the node itself. The suffix tag names list of an XML node contains all the distinct tag names in its corresponding sub-tree, which is described in [2]. For ease of implementation and store efficiency, we present a novel data structure called suffix bitmap to compress suffix tag names list. Suffix bitmap contains the non-structural information of XML sub-tree. As bitwise computation can be processed efficiently, we can skip most of the unmatched subtrees using bitwise suffix bitmap comparison. Therefore, the suffix bitmap can be deployed to filter the unmatched subtrees of XML documents.

In this paper, we will integrate suffix bitmap filtering into the traversal matching algorithm and the structural join matching algorithm. The experiments show that the extended matching algorithms considerably outperform original algorithms. Also, we present the construction procedure of suffix bitmap with linear time complexity. To 
reduce the memory consumption further, we also present the variable-length suffix bitmap.

The rest of the paper is organized as follows. Section 2 describes the suffix bitmap and its construction algorithm. Section 3 integrates the suffix bitmap filtering into original matching algorithms. Section 4 compares the extended matching algorithms to the original algorithms. Section 5 lists some related work.

\section{Suffix Bitmap}

\subsection{Global Order of Tag Names}

Given a XML document, we define the global order of tag names. Each distinct tag name has a global sequence number, which is an incremental positive number and starts at 0 . The assigned function is:

\section{Definition 1}

$\gamma:$ tagName $\rightarrow$ GSN GSN is increasing numeric that starts from 0

$G S N_{\text {tagname } 1}<G S N_{\text {tagname2 }}$ iff tagname1 first appears before tagname2 on XML document import After global sequence numbers assigning, we get the global order of tag names. We represent the global order relation of tag names as an order set called tag names set.

\section{Definition 2}

Set $_{\text {tagname }}=\left\{\right.$ tagname $_{0}$, tagname $_{1}, \ldots$, tagname $\left._{n-1}\right\}$

The GSN of tagname is $i$

For example, in figure1, tagname is $_{1}$ bib, tagname ta $_{2}$ is book and so on.

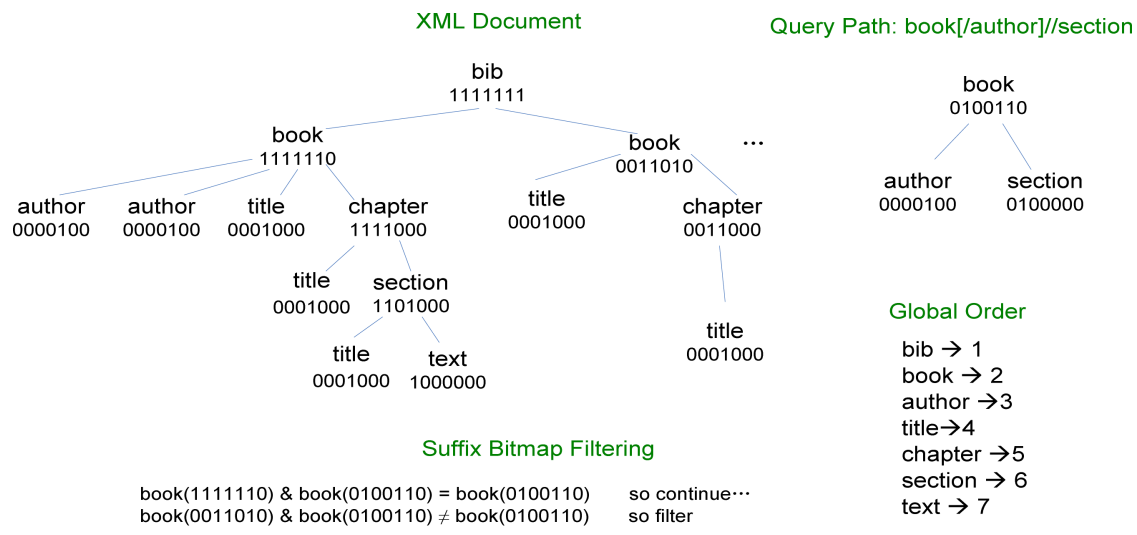

Fig. 1. Suffix Bitmap Filtering

\subsection{Suffix Bitmap}

We first give the description of suffix tag names list. In XML document tree, each node $n$ corresponds to a sub-tree SubTree $_{n}$. The suffix tag names list of node $n$ contains all distinct tag names appeared in $\mathrm{SubTree}_{n}$. Due to the global order of tag 
names, we can represent suffix tag names as a bitmap, called suffix bitmap. We define the suffix bitmap as follows:

\section{Definition3}

Suffix Bitmap is the bitmap format of suffix tag names list. It has $m$ bits.

Here $m$ is the cardinality of Set tagname

Given a bit Bit in suffix bitmap of node $n$

Bit $_{i}= \begin{cases}1 & \text { if tagname } \\ 0 & \text { appears in } \text { SubTree }_{n}\end{cases}$

For example, in figure1, chapter(1111000) has title, chapter, section, text in its subtree. We attach a suffix bitmap to each node in XML document tree. So the memory consumption of total suffix bitmaps is node_count $* m / 8$ bytes, where node_count is the number of nodes in XML document.

\subsection{Construction of the Suffix Bitmap}

We give a preorder construction algorithm of the suffix bitmap, which runs on XML document import. The detail description is below:

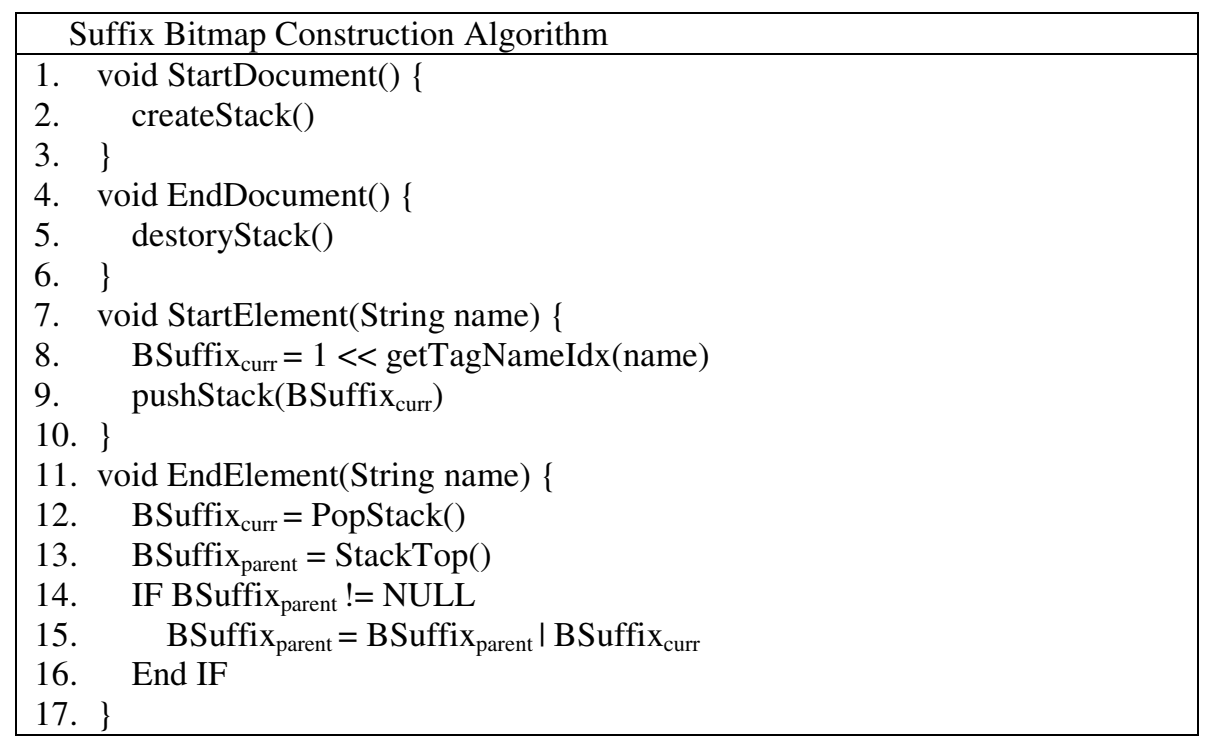

As shown in above algorithm, we use a stack to hold the in-coming suffix bitmap. When StartElement call, we create the corresponding suffix bitmap and push it into the stack. When EndElement call, we pop the corresponding suffix bitmap out and adapt parent node's suffix bitmap by or operation. Thus, the construction order is

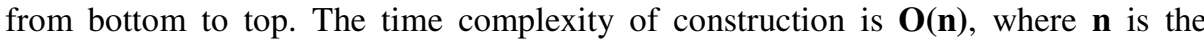
number of nodes in XML document. The function getTagNameIdx inputs a tag name and outputs the corresponding global sequence number.

The readers maybe find a problem in our construction algorithm that is we can't determine the length of suffix bitmap during XML import. There are several methods 
to tackle this problem. One method is we implement the construction of suffix bitmap after XML import. The other method is we pre-estimate the length of suffix bitmap and if the real length of suffix bitmap is larger than the estimate length, we implement the construction again after XML import. Those methods are all inefficient. In the next subsection, we will propose variable-length suffix bitmap to solve this problem.

\subsection{Variable-Length Suffix Bitmap}

Since we can't determine the final cardinality of Set tagname, the variable-length suffix

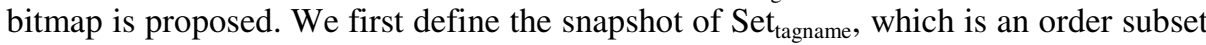
of Set tagname, as follows:

\section{Definition4}

Snapshot $_{k}=\left\{\right.$ tagname $_{i} \mid i \leq k$, tagname $_{i} \in$ Set $\left._{\text {tagname }}\right\}$

Set tagname has $m$ Snapshots on xml document import, where $m$ is the cardinality of Set tagname

The variable-length suffix bitmap has the initial length that is equal to the cardinality of the snapshot ${ }_{k}$ at the moment of creation. When the children suffix bitmap modify the parent suffix bitmap, the length of variable-length parent suffix bitmap is adaptive to the cardinality of current snapshot ${ }_{\mathrm{k}}$. Thus, we modify the length of suffix bitmap in line 8 and 15 of above procedure to corresponding snapshot ${ }_{\mathrm{k}}$ cardinality.

Because the global sequence number of each distinct tag name is invariable since creating, the variable-length suffix bitmap has the same effect as the fix-length suffix bitmap, except that we need to fill the variable-length suffix bitmap with 0s bits during the structural matching, which will be described in next section.

For some $\mathrm{xml}$ documents that contain many distinct tag names, our fixed-length suffix bitmap may be waste of large space. We can solve this problem using the variable-length suffix bitmap. To save space further, we present frequent query tag names to reduce the suffix bitmap, which employs some policies of data mining on $\mathrm{xml}$ queries.

\section{Structural Matching Filtering}

\subsection{Traversal Filtering}

The suffix bitmap contains the tag names information of each sub-tree, so it can judge the unmatched subtrees. Given a query path $P$ and a node $n_{p}$ on $P$, the suffix bitmap of query node is the same as the suffix bitmap of the document node. We have the filtering formula as follows:

\section{Formula 1}

Given the suffix bitmap BSuffix of element $n$, and the suffix bitmap QSuffix of query node $n_{p}$ IF BSuffix \& QSuffix $\neq$ Qsuffix

Then there must be no matching occurrence in Subtree ${ }_{n}$

In the formula 1, QSuffix must be the fix-length suffix bitmap, and BSuffix may be the variable-length suffix bitmap. However, it doesn't impact the correctness of the formula 1. For example, in figure 1, we filter xpath book[/title]//section. The QSuffix 
of path root book is 0100110. The element book(0011010) is filtered because it satisfies formula 1 .

Based on the formula 1, we extend the classical traversal matching algorithm and integrate the suffix bitmap filtering into it. During matching traverse, we can skip most of the unmatched sub-tree by employing the formula 1 . We give the modification detail of DFS algorithm, which is a state-of-the-art traversal algorithm.

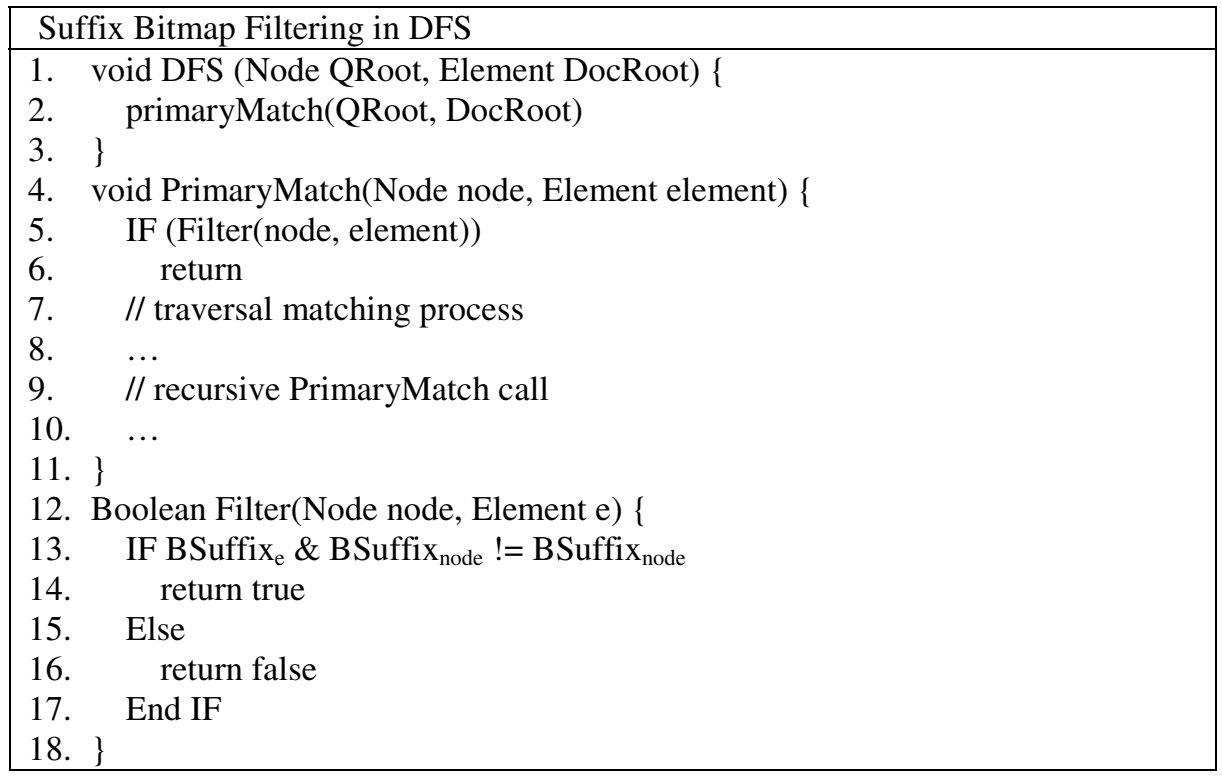

As shown in above algorithm, we call Filter function before each node matching. If the node satisfies the filtering condition, we skip the following matching process and recursive PrimaryMatch call again. Therefore, the bitmap suffix filtering is a highly efficient technique to skip most of the unmatched sub-trees. The efficiency of speedup is dependant on hit ratio of the structural matching. If hit ratio of the structural matching is fairly low, the filtering will be highly efficient. Since the cost of the function Filter is tiny, it adds very low overhead to matching process.

\subsection{Cursor Stream Filtering}

The structural join algorithms based on numbering schema are also the classical matching algorithms, which fall into the set-based category. The structural join algorithms utilize the structural numbering to judge the relationship of candidate nodes, which are obtained by the stream cursor. A disadvantage of structural join algorithms is that there often exist many useless intermediate results during processing because of uncertainty. Using the suffix bitmap filtering, we can eliminate most of useless intermediate results, therefore speed up the matching in structural join algorithms. The extra portion about integrating the suffix bitmap filtering into the structural join is described below: 


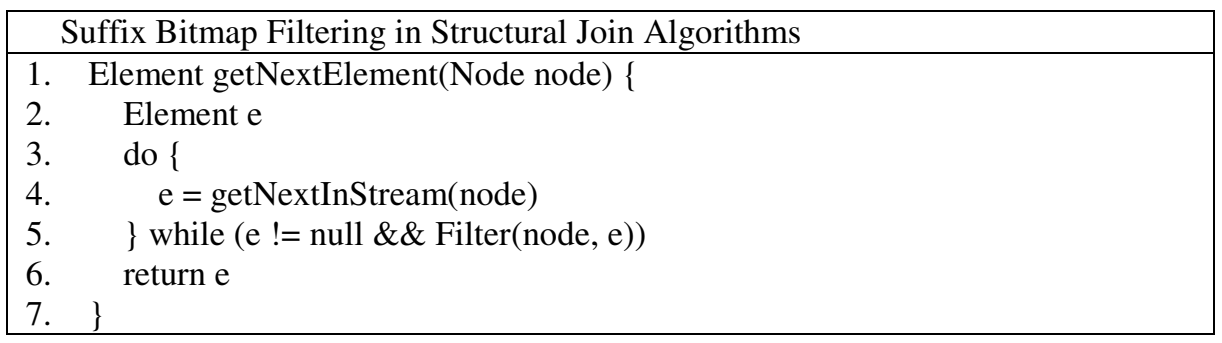

As shown in pseudo-code, the function getNextElement is the basic function of cursor operation of structural join. We encapsulate it to a loop style, which skip the unmatched elements by the suffix bitmap filtering that has been described in above subsection. It can save costly time to filter the unmatched nodes. There has one difference between the filtering in traversal algorithms and the filtering in structural join algorithms. The former is to skip the unmatched sub-trees and the latter is to skip the unmatched nodes. However, there has the same effect that is the speedup.

\section{Experiments}

In this section, we present results and analyses of experiments on the comparison between extended algorithms and previous algorithms. All of our experiments were performed on a PC with Pentium4 2.4GHz CPU, 1GB memory and 120 GB IDE hard disk. The OS is Windows XP. We implemented all algorithms in JAVA. We implemented DFS, FS-DFS, SJoin and FS-SJoin algorithms. DFS is a depth-first traversal algorithm with inline predication checking. SJoin is a novel bottom-up structural join algorithm that enhances the holistic stack join algorithm. FS-DFS is our extended DFS algorithm with the suffix bitmap filtering. And FS-SJoin is our extended SJoin algorithm with the suffix bitmap filtering. The datasets include Nasa, XMark and Treebank [8]. There have three groups of queries: QN, QX and QT which correspond to Nasa, XMark and Treebank. The detail of datasets and queries is below:

Table 1. Datasets

\begin{tabular}{lllll}
\hline Name & Type & Size(MB) & Elements & Level \\
\hline Nasa & Small & 24.5 & 476,646 & 8 \\
XMark & Medium & 113.8 & $1,666,315$ & 12 \\
Treebank & Large & 84 & $2,437,666$ & 36 \\
\hline
\end{tabular}

We tested the performance of all algorithms by running each group of queries 1000 times. For the purpose of simplicity, we ran test codes all in memory and didn't refer to I/O. The figure 2 depicts the comparison of algorithm performance. As shown in figure 2, we find that our extended algorithms outperform previous algorithms $10 \% \sim 300 \%$. SJoin outperforms DFS when the query has many ancestor-descendant edges. DFS outperforms SJoin when the query has many parent-child edges. 
Table 2. Queries

\begin{tabular}{ccc}
\hline QID & Results & Query \\
\hline QN1 & 2407 & reference//source[/journal]//date \\
QN2 & 60663 & tableHead[//tableLink/title]//name \\
QN3 & 23224 & /datasets//fields[//definition]//para \\
QN4 & 9001 & reference//journal[/title][/name]//author \\
QN5 & 813 & dataset[/keywords]/reference[/related]/source/journal/name \\
QN6 & 13122 & tableHead//footnote \\
QX1 & 1629 & /site/people/person[/profile[age]/education]/phone \\
QX2 & 3274 & /site/people/person[//age]//education \\
QX3 & 6409 & people[//education]//age \\
QX4 & 6285 & people//address[//city]//province \\
QX5 & 6000 & europe/item[/name]/description \\
QX6 & 18579 & closed_auction[//date]//annotation//text \\
QT1 & 3 & VP[/DT]//PRP_DOLLAR_ \\
QT2 & 151 & S/VP/PP[/NP/VBN]/IN \\
QT3 & 16 & S[//MD]//ADJ \\
QT4 & 5 & S[/JJ]/NP \\
QT5 & 31 & NP[//RBR_OR_JJR]//PP \\
QT6 & 21 & NP/PP[//NNS_OR_NN]//NN \\
\hline
\end{tabular}
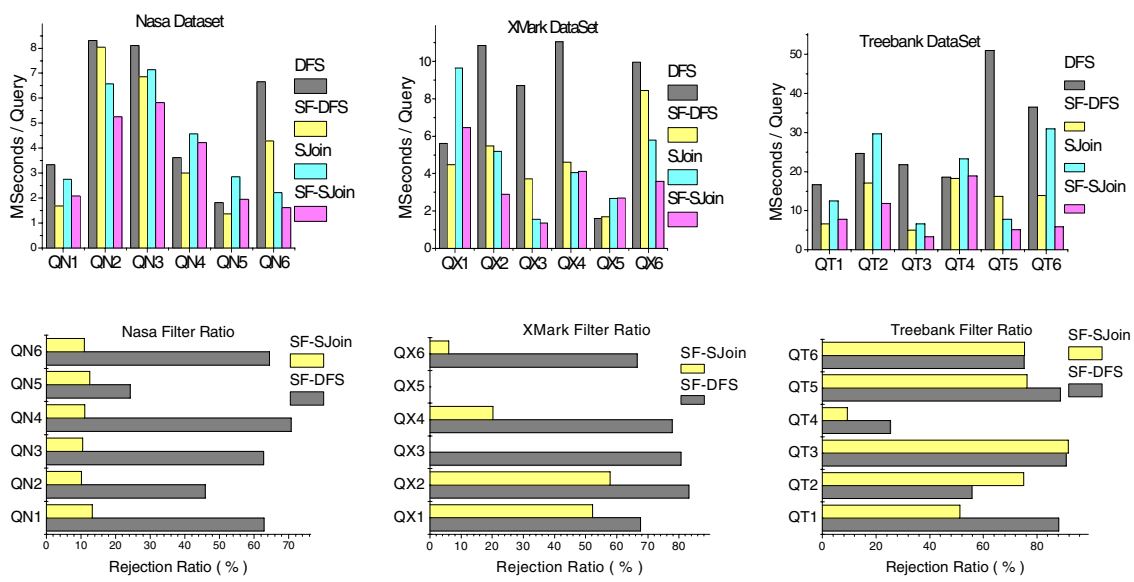

Fig. 2. Query Time and Filter Ratio

However, SF-DFS outperforms DFS at all queries. SF-SJoin outperforms SJoin as well. Also, figure 2 depicts the filter ratio about these three groups of queries. It shows that the efficiency of speedup depends on the filter ratio. When the filter ratio is higher, the efficiency of speedup is more desirable. We can find that the filtering adds very low overhead to matching process when the filter ratio is zero, which is shown in QX5. 


\section{Related Work}

There has been much work in the area of xml structural matching. Structural join [1] and twig join [3] are efficient structural matching algorithms that have been extensively studied. These algorithms determine structural relationship of any two nodes by employing numbering schemes that have also been studied. DFS and BFS [7] are classical traversal algorithms for XML structural matching, which are extensively applied to xml summary graphs [5].

Also, there has been much previous work on speedup of xml structural matching. XR-Tree [4] and XB-Tree [3] are two index strategies to accelerate structural join. [6] proposed a look-ahead approach to skip useless intermediate results in holistic join. Recently, the Subtree Label is presented by [2] in native xml storage system. The Subtree Label is similar to our suffix bitmap, yet doesn't refer to the compression.

\section{Conclusions and Future Work}

In this paper, we present a novel filtering technology for structural matching, which we called suffix bitmap filtering. Based on the suffix bitmap filtering, we extend two state-of-the-art structural matching algorithms: the traversal matching algorithm and the structural join matching algorithm. For the traversal matching algorithm, we skip most of unmatched sub-trees in terms of the suffix bitmap comparison. For the structural join matching algorithm, we skip most of unmatched candidates as well. The experiments show that our extended algorithms perform significantly better than the previous algorithms, especially when the hit ratio of the matching is low.

In future, we will study the suffix bitmap of frequent tag names. Furthermore, the self-tuning technology along with several filtering policies will be considered to speed up the structural matching.

\section{References}

1. Al-Khalifa, S., Jagadish, H.V., Patel, J.M., Wu, Y., Koudas, N., Srivastava, D.: Structural joins: A primitive for efficient XML query pattern matching. ICDE (2002)

2. Boulos, J., Karakashian, S.: A New Design for a Native XML Storage and Indexing Manager. EDBT (2006)

3. Bruno, N., Koudas, N., Srivastava, D.: Holistic twig joins: Optimal XML pattern matching. SIGMOD Conference (2002)

4. Jiang, H., Lu, H., Wang, W., Ooi, B.: XR-Tree: Indexing XML Data for Efficient Structural Joins. ICDE (2003)

5. Kaushik, R., Shenoy, P., Bohannon, P., Gudes, E.: Exploiting Local Similarity for Indexing Paths in Graph-Structured Data. ICDE (2002)

6. Lu, J., Chen, T., Ling, T.: Efficient Processing of XML Twig Patterns with Parent Child Edges: A Look-ahead Approach. Proc of CIKM (2004)

7. Wang, W., Wang, H., Lu, H., Jiang, H., Lin, X., Li, J.: Efficient Processing of XML Path Queries Using the Disk-based F\&B Index. VLDB (2005)

8. XML Data Repository In http://www.cs.washington.edu/research/xmldatasets/ 